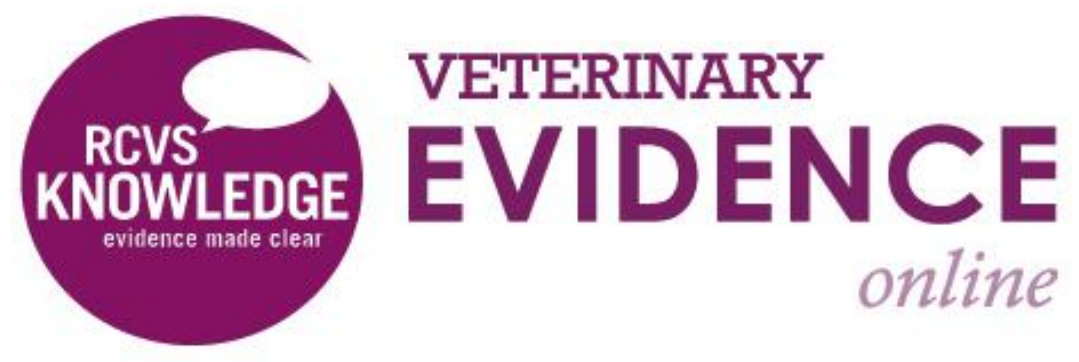

\title{
The Use of Canine Stifle Orthotics for Cranial Cruciate Ligament Insufficiency
}

\section{B.J. Carr DVM, CCRT ${ }^{1}$}

S.O. Canapp Jr. DVM, MS, CCRT, DACVS, DACVSMR ${ }^{1{ }^{*}}$

S. Meilleur DVM, CCRP ${ }^{1}$

S.A. Christopher VMD, CCRP ${ }^{1}$

J. Collins $\mathrm{CO}^{2}$

C. $\operatorname{Cox} \mathrm{MS}^{1}$

\footnotetext{
${ }^{1}$ Veterinary Orthopedic and Sports Medicine, Annapolis Junction, MD, USA

${ }^{2}$ K-9 Orthotics \& Prosthetics Inc., Nova Scotia, Canada

* Corresponding Author (scanapp@vosm.com)
}

ISSN: 2396-9776

Published: 20 Jan 2016

in: Vol 1, Issue 1

DOI: http://dx.doi.org/10.18849/ve.v1i1.10.g21

Reviewed by: Wanda Gordon- Evans DVM PhD DACVS DACVSMR Adam Swallow BVSC MRCVS 
Objective: To assess weight bearing of dogs treated for unilateral cranial cruciate ligament insufficiency with a custom stifle orthotic.

Background: Cranial cruciate ligament (CCL) insufficiency is the most common cause of hind limb lameness in dogs. While there are numerous options for surgical management, surgery is not always an option. Recently, the use of canine stifle orthotics has also emerged as a means to non-surgically manage patients with cranial cruciate ligament insufficiency.

Evidentiary value: This is a retrospective study of ten dogs treated for unilateral cranial cruciate ligament rupture with a stifle orthotic.

Methods: Medical records (January 2005- December 2012) of ten dogs treated for unilateral cranial cruciate ligament rupture with a stifle orthotic were reviewed. Temporospatial gait analysis was performed using a pressure sensing walkway at baseline and 90 days or greater post orthotic placement to identify weight bearing with total pressure index \% (TPI\%).

Results: TPI\% improved significantly by $5.1 \%$ in the affected limb when compared to baseline $(p=0.0020)$. At final gait analysis, TPI\% significantly improved by $3 \%$ in the affected limb with the orthotic off when compared to the unaffected $\operatorname{limb}(p=0.0020)$.

Conclusion: Custom canine stifle orthotics allow for improved weight bearing in the affected limb.

Application: Custom canine stifle orthotics should be considered for cases with concurrent medical conditions or financial constraints that do not allow for surgical intervention.

Abbreviations: TPI\%: Total Pressure Index Percentage

\section{INTRODUCTION}

Cranial cruciate ligament (CCL) insufficiency is the most common cause of hind limb lameness in dogs (1). Many recent studies have shown surgical stabilization to be effective in managing cranial cruciate ligament insufficiency (2-12). While there are numerous options for surgical management, surgery is not always an option. Concurrent medical problems, financial concerns, or staging procedures for bilateral CCL insufficiency are all reasons for either forgoing or delaying surgical treatment. One recent study reported that dogs treated surgically and with non-surgical methods (weight loss, administration of NSAIDs, and rehabilitation therapy) had better overall outcomes compared with dogs that were treated via non-surgical methods alone (6). Recently, the use of canine stifle orthotics has also emerged as a means to non-surgically manage patients with cranial cruciate ligament insufficiency.

In human medicine, knee braces are commonly used for multiple conditions (13-19). Bracing of the human knee has been shown to enhance proprioception/joint position sense, permit the injured limb to relax, reduce fatigue in injured limb, provide some mechanical protection against impact, and slow movement down to 
allow muscles time to react and control motion (14,17-22). Recently there has been debate over the efficacy of functional knee braces in neutralizing excessive anteroposterior translation in the ACL deficient knee. Functional knee braces are designed to reduce knee instability by neutralizing forces to support the affected ligament (i.e. neutralizing cranial tibial thrust in the $\mathrm{ACL}$ deficient knee). One recent review reported that biomechanical evaluations of functional braces have been mixed and concluded further functional brace studies designed to apply forces to the knee joint similar in magnitude to the native ACL should be performed (16). In spite of this, functional knee braces are still thought to be effective in controlling anteroposterior translation in ACL-deficient knees under low loading conditions (17-22). However, knee braces are not believed to be effective under high loading conditions, such as those that occur during athletic activity $(16,17,20,21)$.

Functional knee braces are utilized in veterinary medicine with the most common indication being cranial cruciate ligament insufficiency (23). To the author's knowledge, no objective data is available to support the use of stifle orthotics in dogs. The goal of this study is to evaluate weight bearing of patients diagnosed with a unilateral cranial cruciate ligament insufficiency while wearing a stifle orthotic using a pressure sensing walkway. The hypothesis is that the use of a custom stifle orthotic would allow for increased weight bearing as demonstrated by temporospatial gait analysis using a pressure sensing walkway.

\section{RESULTS}

\section{Patient Demographics}

Of the 160 patients that have been treated at VOSM with stifle orthotics for CCL insufficiency, 10 fit the inclusion criteria for this study. All 10 patients were assessed at baseline and presented 90 days or greater post orthotic placement for re-evaluation (mean, 180 days; range $90-540$ days). There were 9 breeds represented, the Labrador retriever was the most common $(n=2,20 \%)$, followed by one each of Beagle, Bernese Mountain Dog, Doberman, German Shorthaired Pointer, Mastiff, Newfoundland, Pit Bull Terrier and West Highland White Terrier. The average age was 10.4 years old (range 3-15.7 years, median 10.4 years). The average weight was $29.8 \mathrm{~kg}$ (range 6.8-52.7kg, median 31.8kg). Males accounted for 1 patient (10\%) who was altered. Females accounted for 9 patients (90\%) with 8 being altered and 1 being intact (Table 1 and Table 2).

\section{Medications:}

Seven of the ten patients were on medications when they presented at 90 days or greater post orthotic placement. Three of the seven were taking gabapentin and tramadol for pain. Five of the ten patients were taking a non-steroidal anti-inflammatory medication (carprofen). Two patients were on joint supplements, including Adequan(1), Dasuquin with MSM(1). One patient with concurrent hepatic insufficiency was on Denamarin and meclizine. Many patients were on a multimodal drug therapy plan (Table 3).

\section{Orthopedic Examination Findings:}

Of the ten study patients identified, the right stifle was affected in $50 \%$ of the cases and the left stifle affected in $50 \%$ cases. Patients with bilaterally affected stifles were excluded from the study. At diagnosis cranial drawer in both flexion and extension was identified in 8 patients $(80 \%)$ and 2 patients $(20 \%)$ had cranial drawer in flexion only. The meniscus was evaluated in all cases with only $1(10 \%)$ having a suspect meniscal injury as identified by a meniscal click, pain on deep flexion, and positive McMurray test for meniscal injury (Table 2). No examination findings had changed at the 90 days or greater post orthotic placement evaluation. No evidence of neoplasia or progression of osteoarthritis was noted on radiographs of the affected stifle. 


\section{Total Pressure Index}

Baseline data showed an average total pressure index (TPI\%) of 21.6\% (range 14.6\%-25.7\%) in the unaffected limb and $13.4 \%$ (range $7.3 \%-18.8 \%$ ) in the affected limb. At 90 days or greater post orthotic placement, when dogs were walked over the pressure mat without their orthotic in place, an average TPI\% of $22.1 \%$ (range $19 \%$ 25.3\%) in the unaffected limb and $15.5 \%$ (range 11.7\%-18.9\%) in the affected limb was found (Fig $1 ; p=$ 0.0020). There was also a statistically significant increase in affected limb TPI\% without the orthotic when compared to baseline data (Fig 2; $p=0.0195$ ). When dogs were walked over the pressure mat with their orthotic in place, an average TPI\% of $21.3 \%$ (range $17.7 \%-24.9 \%$ ) in the unaffected limb and $18.5 \%$ (range 13.9\%-22.1\%) in the affected limb was found (Fig 3; $p=0.0098$ ). When comparing the patient using their orthotic to their initial consultation, we found a statistically significant average increase in TPI \% of $5.1 \%$ in the affected limb (Fig 2 and Fig 4; $p=0.0020$ ). When comparing the difference of wearing the orthotic versus not wearing the orthotic on the patients final recheck analysis, we found a statistically significant average increase of $3 \%$ in the affected limb (Fig 2 and Fig $4 ; p=0.0020$ ).

\section{Validated Client Survey}

On 90 days or greater post orthotic placement, owners were asked to complete the Helsinki Chronic Pain Index. Only five of the ten owners completed the survey. Owners were asked to assess the patient's mood, pain level, willingness to play, and ability to walk, trot, gallop, jump, rise, move after periods of long rest, and move after heavy exercise. Client follow-up (mean, 180 days; range 90 - 540 days) revealed overall improvement in terms of function in all cases, indicating that patients were able to return to near normal (prior to stifle instability) activity levels. While only five of the ten owners completed the survey, all owners expressed satisfaction with the orthotic. Only two of the owners stated the orthotic required minor adjustments after the initial placement and fitting appointment.

\section{DISCUSSION}

The present study demonstrates that custom canine stifle orthotics allow for improved weight bearing in patients with unilateral cranial cruciate ligament insufficiency. Validated client survey also revealed overall improvement in terms of function. Thus, canine stifle orthotics should be considered for those cases with concurrent medical conditions or financial constraints that do not allow for or require a delay of surgical intervention.

While surgery is the gold standard treatment for treating cranial cruciate ligament insufficiency, this study showed that custom stifle orthotics can still help improve overall function. In the one case where a meniscal click, pain on deep flexion, and positive McMurray test for meniscal injury was identified, this patient also had improved weight bearing while wearing the stifle orthotic. Further study would be needed to fully assess the outcome of custom stifle orthotics in dogs with concurrent meniscal injury. Whether surgery is declined for medical or financial reason, custom stifle orthotics can be offered to help improve function. However, in the authors' experience, prior to recommending a custom stifle orthotic, it is important to consider patient and owner factors as complications can include rub sores, persistent instability, and secondary soft tissue injuries if the orthotics are not casted for, fitted, worn and monitored correctly and consistently. 
The effect of custom stifle orthotics combined with rehabilitation therapy remains unknown as all patients in this study declined enrollment in a dedicated rehabilitation therapy program. It is possible that rehabilitation therapy could further improve function as previous studies have shown that rehabilitation therapy alone can improve patient outcome (6). Future study should assess the outcome and level of return to function of patients with cranial cruciate ligament insufficiency treated with rehabilitation therapy and a custom stifle orthotic.

It is also important to note that custom stifle orthotics have been used for a variety of conditions, including collateral ligament injuries, deranged stifles, post stem cell therapy, and post patellar ligament repair (23). While assessing these outcomes is beyond the scope of this study, future studies should also assess outcomes of patients treated with a custom stifle orthotic for such conditions.

Limitations of this study are predominantly due to the small sample size and retrospective nature of the study. Many of the initial 160 patients identified were either deceased or lost to follow up at the time the study was performed. Also, all patients were not reassessed at 90 days following stifle orthotic placement (range $90-$ 540 days), and there was no control group. Seven of the ten patients were also on medications over the course of the study, and this could also affect outcomes. Further study is still needed to assess the efficacy of canine stifle orthotics. Also, while improved weight bearing may suggest improved comfort, this study does not assess the kinetic or kinematic effect of custom stifle orthotics on the stifle or gait. While stifle orthotics are believed to limit anterior tibial translation and excessive internal rotation, further study is indicated to assess this.

While further study is still needed to assess the kinetic and kinematic effect of custom stifle orthotics, this study demonstrates that custom canine stifle orthotics allow for improved weight bearing on objective gait analysis and satisfaction in terms of function by validated client survey. Thus, custom stifle braces should be considered for those cases with concurrent medical conditions or financial constraints that do not allow for or require a delay of surgical intervention.

\section{METHODS \& MATERIALS}

\section{Patient Selection}

Medical records (January 2005- December 2012) of Veterinary Orthopedic and Sports Medicine (VOSM) of dogs treated for cranial cruciate ligament rupture with a stifle orthotic were reviewed. Inclusion criteria were a diagnosis of a unilateral cranial cruciate ligament rupture on the basis of positive cranial drawer and cranial tibial thrust, a baseline temporospatial gait analysis at the time of diagnosis, radiographs of the affected stifle at the time of diagnosis, and stabilization with a custom stifle orthotic with a polycentric mechanical joint and harness system for suspension. ${ }^{a}$ All patients included in the study were fitted for the stifle orthotic with their owner present. At the time of the stifle orthotic fitting, all owners were shown how to correctly apply the orthotic and instructed to apply the orthotic when the patient was active. Initially dogs were placed in the brace for 2 to 4 hours per day during periods of activity with the owner checking the orthotic every 30 to 60 minutes to ensure proper fit. This time was gradually increased over 2 weeks until the patient was wearing the orthotic all day during periods of activity. Owners were allowed to remove the orthotic at night while the patient was sleeping. 
Dogs with concurrent orthopedic disease, bilateral cranial cruciate ligament rupture, or concurrent neurologic disease were excluded from the study. Dogs who went on to have surgical repair for the cranial cruciate ligament instability, were deceased, or lost to follow up were also excluded from the study. Finally, if the recommendations for orthotic usage were not complied with, the dog was excluded from the study. Owners of dogs who met the study inclusion criteria were contacted and invited to bring their dog back for a 90 days or greater post orthotic placement re-evaluation that included an orthopedic evaluation, objective gait analysis, and validated client survey.

\section{Orthopedic Evaluation}

Patients were asked to return to VOSM for an evaluation 90 days or greater post orthotic placement. A complete orthopedic and neurological examination was completed by the primary investigator. Routine craniocaudal and mediolateral radiographs of the affected stifle were also performed and evaluated by the primary investigator for progression of osteoarthritis and to rule out neoplasia.

\section{Objective Gait Analysis}

Objective gait analysis was performed in a quiet room using a temporospatial pressure sensing walkway at the time of diagnosis and at 90 days or greater post orthotic placement. The walkway system was equipped with an $8.23 \times 0.85-\mathrm{m}$ portable mat that had 29,952 encapsulated sensors. ${ }^{b}$ The active dimensions of the mat were $8.04 \times 0.61-\mathrm{m}$. A $1.25 \times 0.85-\mathrm{m}$ section of inactive mat was placed at each end of the walkway system to provide transition surfaces when entering and exiting the system. The mat was calibrated by the manufacturer. The walkway system interfaced with a computer and software program for processing and storage of raw data recorded from quadruped gait analysis. ${ }^{c}$ One camera was positioned at a height of $50 \mathrm{~cm}$ at the end of the walkway system to record movement. ${ }^{d}$ Digital video files of each pass across the walkway system were automatically linked to the data files for footfall verification. Owners and other dogs were not permitted to be present during gait analysis. Every dog was handled by the same examiner and allowed to acclimate to the room prior to data collection. Dogs were walked on the mat until they appeared relaxed (approximately 4 to 6 passes/dog).

At 90 days or greater post orthotic placement patients were walked down the pressure sensing walkway with the orthotic on and off to collect the total pressure index percentage (TPI\%). Each patient performed three satisfactory passages at a walk with the orthotic on and then the orthotic off. A walk is defined as a 4-beat gait in which all legs step sequentially. A passage down the walkway was considered satisfactory if the patient was walking in a straight line over the center of the mat, looking straight ahead and with less than two standard deviations in variation of the velocity with walk velocity of 0.9 to $1.2 \mathrm{~m} / \mathrm{s}$. A successful passage must consist of at least three full gait cycles consecutively without a change in footfall pattern. The gait analysis data with the orthotic off and with the orthotic on were completed at 90 days or greater post orthotic placement and was compared to baseline gait analysis performed at the time of diagnosis.

\section{Validated Client Survey}

Patient owners completed the Helsinki Chronic Pain Index (HCPI) at 90 days or greater post orthotic placement or were contacted by phone if their dog was no longer an active patient at VOSM. The Helsinki Chronic Pain Index (HCPI) is a validated, reliability tested and responsiveness tested pain questionnaire that was completed by the dog owner. 


\section{Statistical Analysis}

Wilcoxon matched-pairs signed rank test (non-parametric, paired t-test) was performed for all data. ${ }^{e}$ Significance was established at $p<0.05$.

\section{FOOTNOTES}

a. K-9 Orthotics and Prosthetics Inc., Nova Scotia, Canada

b. GaitFour Pressure Sensing Walkway, platinum version, CIR Systems Inc, Harvertown, PA

c. GaitFour, platinum version, CIR Systems Inc, Harvertown, PA

d. Logitech mega pixel web camera, Logitech, Freemont, CA

e. GraphPad Prism version 6.00, GraphPad Software, La Jolla, California USA

1. Witsberger,T. H. et al (2008) Prevalence of and risk factors for hip dysplasia and cranial cruciate ligament deficiency in dogs. Journal of the American Veterinary Medical Association. 232(12) pp 18181824 http://dx.doi.org/10.2460/javma.232.12.1818

2. Kim, S.E. , Lewis, D.D. and Pozzi, A. (2012) Effect of Tibial Plateau Leveling Osteotomy on Femorotibial Subluxation: In Vivo Analysis during Standing. Veterinary Surgery, 41(4) pp 465470http://dx.doi.org/10.1111/j.1532-950X.2012.00973.x

3. Nelson, S.A. et al (2012) Long-Term Functional Outcome of Tibial Plateau Leveling Osteotomy Versus Extracapsular Repair in a Heterogeneous Population of Dogs. Veterinary Surgery. 42(1) pp3850http://dx.doi.org/10.1111/j.1532-950X.2012.01052.x

4. Christopher, S.A. , Beetem, J., and Cook, J.L (21012). Comparison of Long-Term Outcomes Associated With Three Surgical Techniques for Treatment of Cranial Cruciate Ligament Disease in Dogs. Veterinary Surgery. 42(3) pp 329-334

http://dx.doi.org/10.1111/j.1532-950X.2013.12001.x

5. Biskup, J.J etc al (2014) Ability of the Tightrope and Percutaneous Lateral Fabellar Suture Techniques to Control Cranial Tibial Translation. Veterinary Surgery 43(8) pp 959-985 http://dx.doi.org/10.1111/j.1532-950X.2014.12111.x

6. Wucherer, K.L et al (2013) Short-term and long-term outcomes for overweight dogs with cranial cruciate ligament rupture treated surgically or nonsurgically. Journal of the American Veterinary Medical Association. 242(10) pp 1364-1372 http://dx.doi.org/10.2460/javma.242.10.1364

7. Molsa,S.H. et al (2014) Long-term functional outcome after surgical repair of cranial cruciate ligament disease in dogs. BMC Veterinary Research. 10:266 http://dx.doi.org/10.1186/s12917-014-0266-8

8. Nelson, S.A. et al (2013) Long term functional outcome of tibial plateau leveling osteotomy versus extracapsular repair in a heterogeneous population of dogs. Veterinary Surgery $42(1)$ pp:3850http://dx.doi.org/10.1111/j.1532-950X.2012.01052.x

9. Cook, J.L, et al, (2010) Clinical comparison of a novel extracapsular stabilization procedure and tibial plateau leveling osteotomy for treatment of cranial cruciate ligament deficiency in dogs, Veterinary Surgery 39(3) pp 315-323 http://dx.doi.org/10.1111/j.1532-950X.2010.00658.x

10. Headrick, J.F., Zhang, S and Millard, R.P. (2014) Use of an inverse dynamics method to compare the three-dimensional motion of the pelvic limb among clinically normal dogs and dogs with cranial 
cruciate ligament-deficient stifle joints following tibial plateau leveling osteotomy or lateral fabellartibial suture stabilization. American Journal of Veterinary Research. 75(6) pp:554-564

http://dx.doi.org/10.2460/ajvr.75.6.554

11. Au, K.K. , Gordon-Evans, W.J. and Dunning, D (2010) Comparison of short- and long-term function and radiographic osteoarthrosis in dogs after postoperative physical rehabilitation and tibial plateau leveling osteotomy or lateral fabellar suture stabilization. Veterinary Surgery 39(2) pp:173-180 http://dx.doi.org/10.1111/j.1532-950X.2009.00628.x

12. Oxley, B. et al,(2013) Comparison of complication rates and clinical outcome between tibial plateau leveling osteotomy and a modified cranial closing wedge osteotomy for treatment of cranial cruciate ligament disease in dogs. Veterinary Surgery. 42(6): pp 739-750 http://dx.doi.org/10.1111/j.1532950X.2013.12033.x

13. Baltaci, G., Aktas, G., and Camci, E. (2011) The effect of prophylactic knee bracing on performance: balance, proprioception, coordination, and muscular power. Knee Surgery Sports Traumatology Arthroscopy. 19(10) pp1722-1728 http://dx.doi.org/10.1007/s00167-011-1491-3

14. Bodendorfer, B.M. et al, (2013) Anterior Cruciate Ligament Bracing: Evidence in Providing Stability and Preventing Injury or Graft Re-Rupture. The Physician and Sports Medicine. 41(3) pp 92102http://dx.doi.org/10.3810/psm.2013.09.2020

15. Segal, N.A. (2012) Bracing and Orthoses: A Review of Efficacy and Mechanical Effects for Tibiofemoral Osteoarthritis. PM\&R. 4(5) Supplement pp:S89-S96 http://dx.doi.org/10.1016/j.pmrj.2012.01.018

16. Smith, S.D. et al. (2013) Functional bracing of $A C L$ injuries: current state and future directions. Knee Surgery Sports Traumatology Arthroscopy. 22(5) pp 1131-1141 http://dx.doi.org/10.1007/s00167013-2514-z

17. Chew, K.T.L et al, (2007) Current Evidence and Clinical Applications of Therapeutic Knee Braces.American Journal of Physical Medicine \& Rehabilitation. 86(8) pp:678686http://dx.doi.org/10.1097/PHM.0b013e318114e416

18. Birmingham, T.B. et al, (2001) Knee bracing for medial compartment osteoarthritis: effects on proprioception and postural control. Rheumatology. 40(3) pp 285289http://dx.doi.org/10.1093/rheumatology/40.3.285

19. Birmingham, T.B. et al, (2001) Knee bracing after ACL reconstruction: effects on postural control and proprioception. Medicine \& Science in Sports \& Exercise. 33(8) pp 12531258http://dx.doi.org/10.1097/00005768-200108000-00002

20. Giotis, $\mathrm{G}$ et al (2013). Effects of Knee Bracing on Tibial Rotation During High Loading Activities in Anterior Cruciate Ligament-Reconstructed Knees. Arthroscopy. 29(10) pp 16441652 http://dx.doi.org/10.1016/j.arthro.2013.07.258

21. Giotis, $\mathrm{G}$ et al (2011), Knee braces can decrease tibial rotation during pivoting that occurs in high demanding activities. Knee Surgery Sports Traumatology Arthroscopy. 1998) pp 13471354 http://dx.doi.org/10.1007/s00167-011-1454-8

22. Palm, H.G. et al. (2012) Effects of knee bracing on postural control after anterior cruciate ligament rupture. The Knee. 19(5) pp 664-671 http://dx.doi.org/10.1016/j.knee.2011.07.011

23. Canapp, S.O., Campana, D.M and Fair, L.M. (2012) Orthopedic coaptation devices and small-animal prosthetics, In Tobias KM, Johnston SA: Veterinary Surgery Small Animal. St. Louis, Missouri, Elsevier, pp 628-646 
Figure 1: Patient TPI\% at day 90+ Without the Orthotic

\section{TPI\% \\ Brace Off Day 90+}

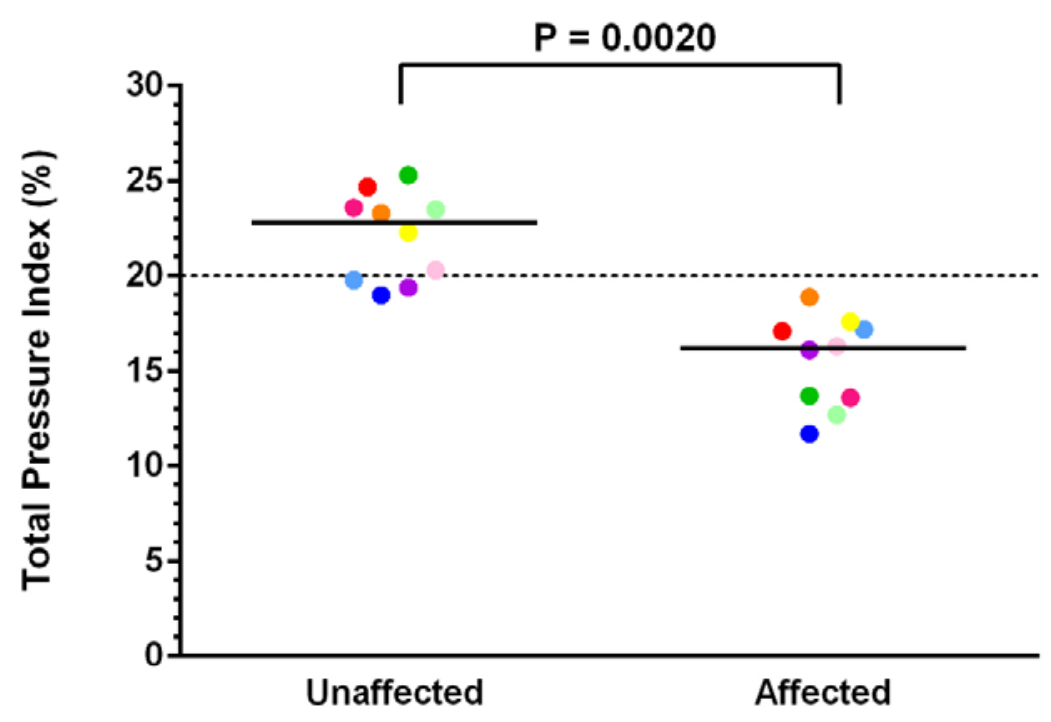

Figure 2: Affected Limb TPI\%

\section{Affected Limb TPI\%}

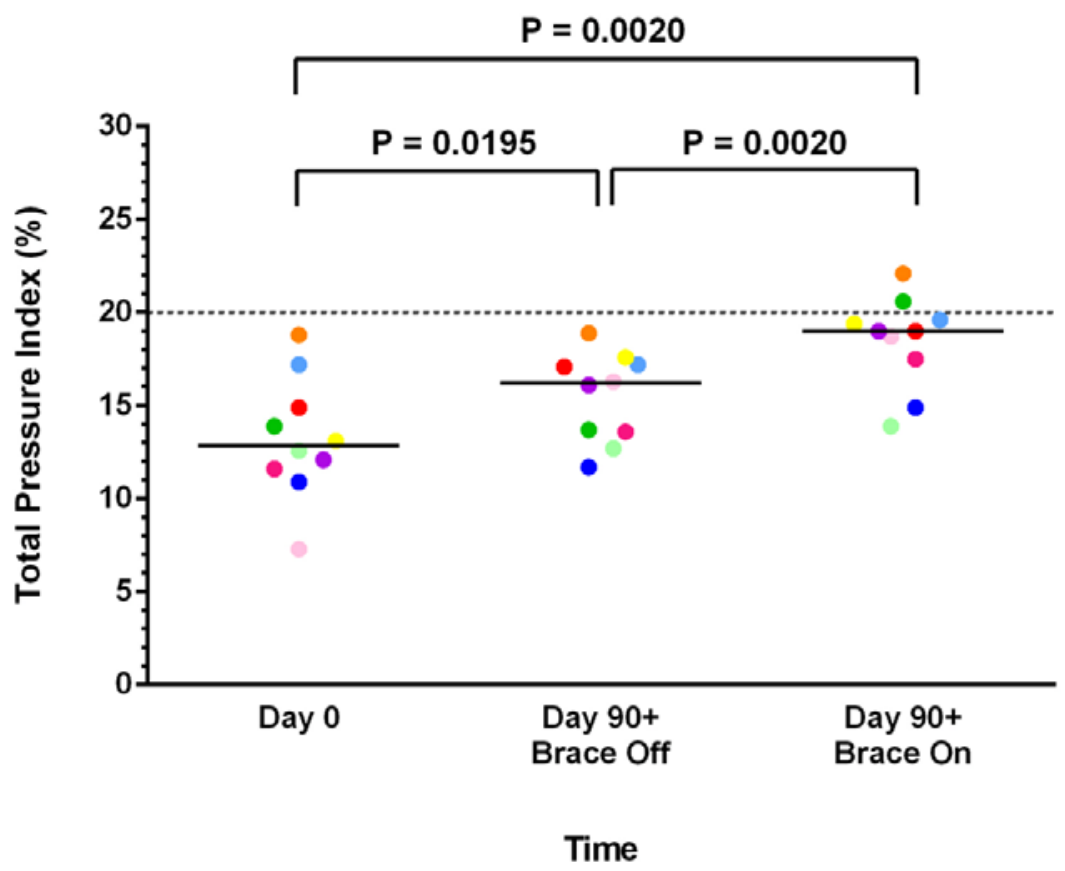


Figure 3: Patient TPI\% at day 90+ With the Orthotic

\section{TPI\% \\ Brace On Day 90+}

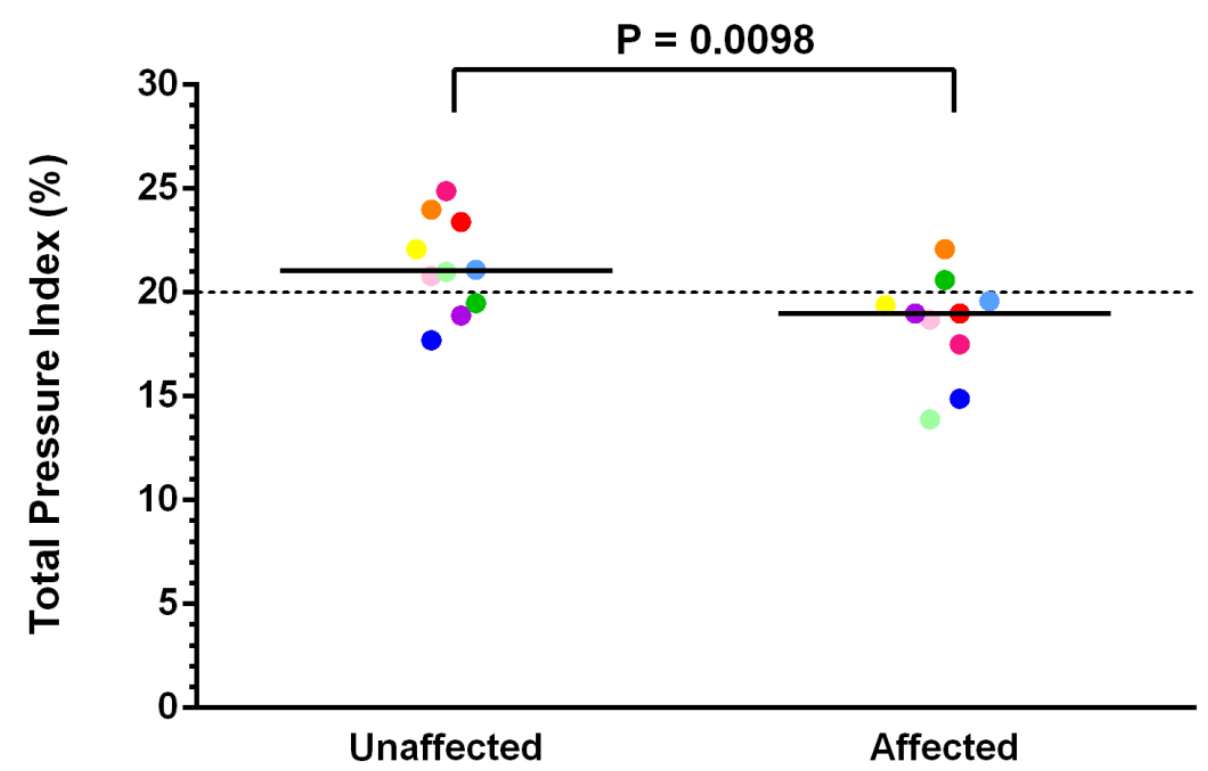

Figure 4: The TPI\% in the Affected Limb of Each Individual Patient Over Time

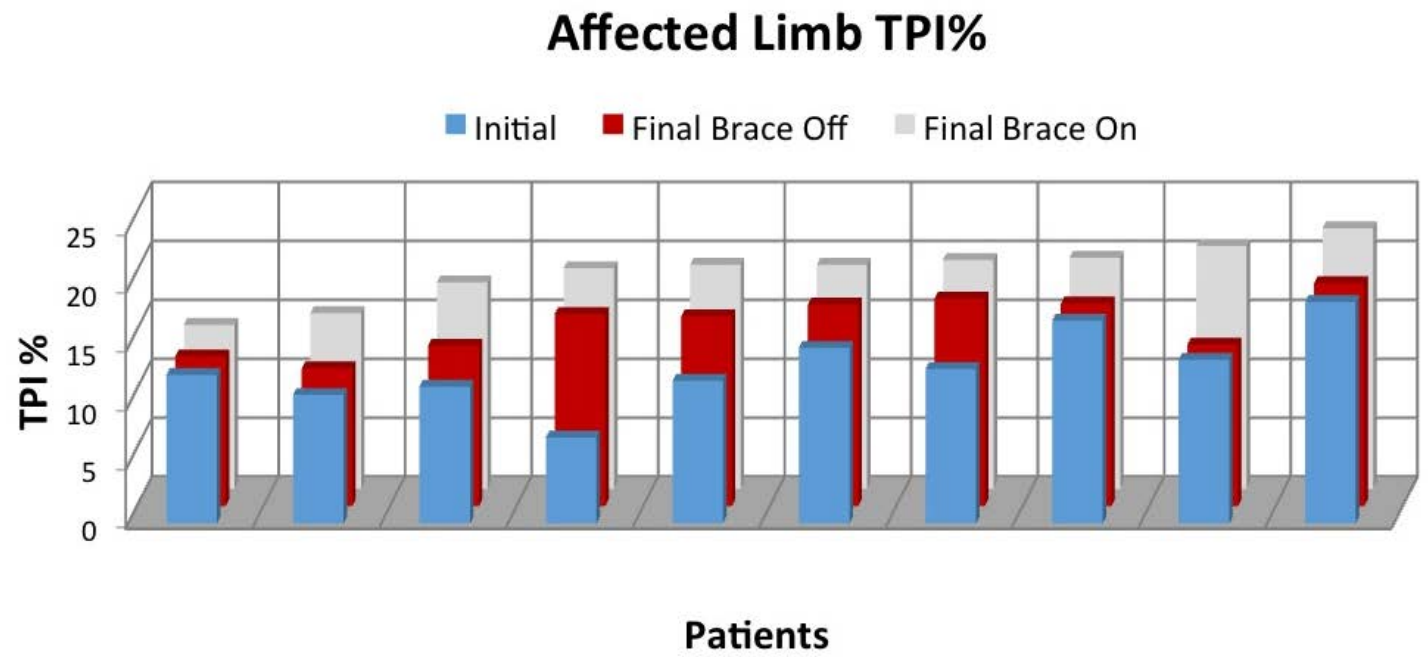


Table 1. Breed Demographics

\begin{tabular}{|l|r|r|}
\hline Breed & \multicolumn{2}{|c|}{$\mathrm{n}=10$} \\
\hline Beagle & 1 & $10.0 \%$ \\
\hline Bernese Mountain Dog & 1 & $10.0 \%$ \\
\hline Doberman & 1 & $10.0 \%$ \\
\hline German Shorthair Pointer & 1 & $10.0 \%$ \\
\hline Labrador Retriever & 2 & $20.0 \%$ \\
\hline Mastiff & 1 & $10.0 \%$ \\
\hline Newfoundland & 1 & $10.0 \%$ \\
\hline Pit Bull Terrier & 1 & $10.0 \%$ \\
\hline West Highland White Terrier & 1 & $10.0 \%$ \\
\hline
\end{tabular}

Table 2. Overall Patient Demographics

\begin{tabular}{|c|c|c|c|}
\hline \multicolumn{2}{|r|}{ Demographics } & \multicolumn{2}{|c|}{$\mathrm{n}=10$} \\
\hline $\begin{array}{l}\text { Average Age } \\
\text { (Range) }\end{array}$ & Years & & $10.4(3-15.7)$ \\
\hline \multirow{2}{*}{$\begin{array}{l}\text { Average Weight } \\
\text { (Range) }\end{array}$} & Lbs & & $65.5(15-116)$ \\
\hline & $\mathrm{Kg}$ & & $29.8(6.8-52.7)$ \\
\hline \multirow{4}{*}{ Gender } & Female, Intact & 1 & $10.0 \%$ \\
\hline & Female, Altered & 8 & $80.0 \%$ \\
\hline & Male, Intact & 0 & $0.0 \%$ \\
\hline & Male, Altered & 1 & $10.0 \%$ \\
\hline \multirow{3}{*}{ Affected Limb } & Left & 5 & $50.0 \%$ \\
\hline & Right & 5 & $50.0 \%$ \\
\hline & Bilateral & 0 & $0.0 \%$ \\
\hline Cause of Instability & Cranial Cruciate Ligament Rupture & 10 & $100 \%$ \\
\hline \multirow{2}{*}{$\begin{array}{c}\text { Cruciate Ligament } \\
\text { Rupture Cases } \\
\text { (n=148) }\end{array}$} & Complete & 8 & $80.0 \%$ \\
\hline & Partial & 2 & $20.0 \%$ \\
\hline \multirow{2}{*}{$\begin{array}{c}\text { Meniscus Tear } \\
(n=138)\end{array}$} & Yes & 1 & $10.0 \%$ \\
\hline & No & 9 & $90.0 \%$ \\
\hline
\end{tabular}


Table 3. Patient Medications

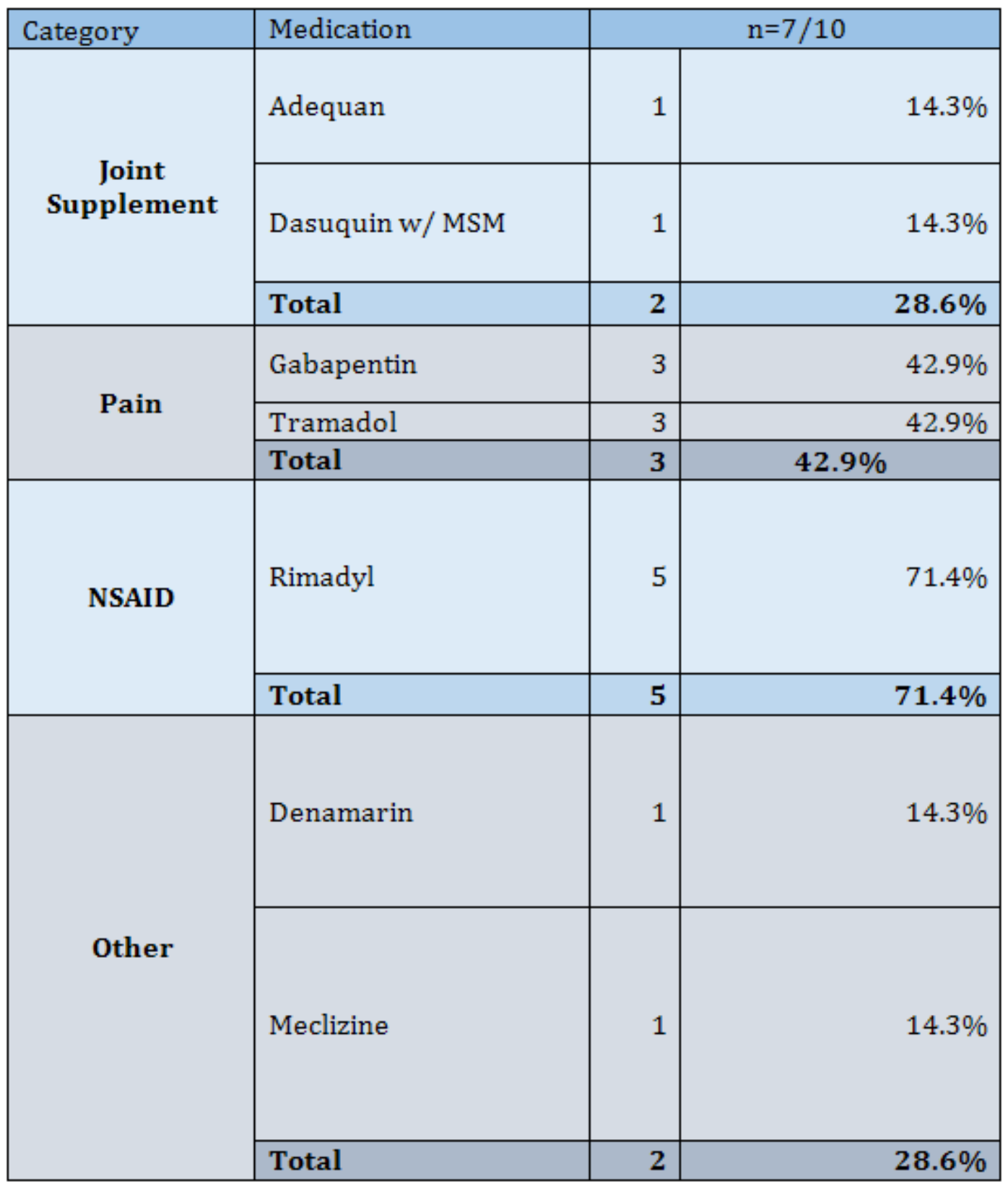




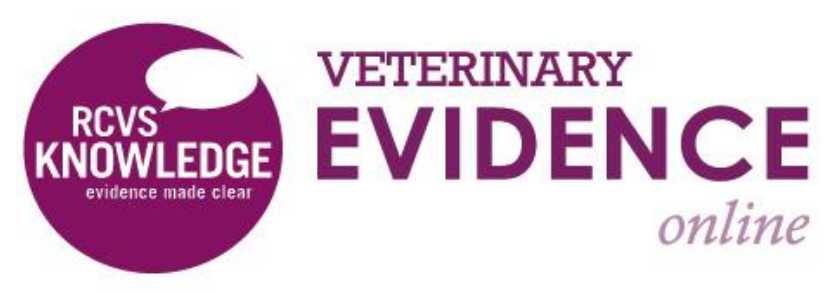

\begin{abstract}
Intellectual Property Rights
Authors of Knowledge Summaries submitted to RCVS Knowledge for publication will retain copyright in their work, but will be required to grant to RCVS Knowledge an exclusive license of the rights of copyright in the materials including but not limited to the right to publish, re-publish, transmit, sell, distribute and otherwise use the materials in all languages and all media throughout the world, and to license or permit others to do so.
\end{abstract}

Authors will be required to complete a license for publication form, and will in return retain certain rights as detailed on the form.

Veterinary Evidence and EBVM Network are RCVS Knowledge initiatives. For more information please contact us at editor@veterinaryevidence.org.

RCVS Knowledge is the independent charity associated with the Royal College of Veterinary Surgeons (RCVS). Our ambition is to become a global intermediary for evidence based veterinary knowledge by providing access to information that is of immediate value to practicing veterinary professionals and directly contributes to evidence based clinical decision-making.

www.veterinaryevidence.org

RCVS Knowledge is a registered Charity No. 230886. Registered as a Company limited by guarantee in England and Wales No. 598443.

Registered Office:

Belgravia House

62-64 Horseferry Road

London SW1P 2AF 